\title{
New low-frequency electromagnetic modes associated with neutral dynamics in partially ionised plasma
}

\author{
A. A. Shaikh ${ }^{1}$ and A. C. Das ${ }^{2}$ \\ ${ }^{1}$ Department of Physics and Astronomy, University of Leicester, Leicester LE1 7RH, UK \\ ${ }^{2}$ Theoretical Physics Division, Physical Research Laboratory, Navrangpura, Ahmedabad-380 009, India \\ Correspondence to: A. A. Shaikh (profabdu@yahoo.com) \\ Received: 27 November 2012 - Revised: 28 February 2013 - Accepted: 23 April 2013 - Published: 28 May 2013
}

\begin{abstract}
We have investigated the low frequency electromagnetic (EM) modes in inhomogeneous, magnetised partially ionised plasma by incorporating neutral dynamics. We have derived a general EM dispersion relation by using a two-fluids magnetohydrodynamics (MHD) model. Our analysis shows that the neutral dynamics is playing an extremely important role in the physics of magnetised partially ionised plasma by giving rise to new kind of EM modes. We found (1) the new instability is linked with compressibility of neutral particles, the collision between neutral and charged species and the relative streaming in hot/cold, inhomogeneous, magnetised partially ionised plasma, (2) and that neutral dynamics is responsible for the modified (complex) inertial effect on magnetic field lines. Its consequences on the propagation characteristics of Alfvén wave and cyclotron frequency are discussed. Furthermore, a new mode similar to the Langmuir mode is reported. Finally, we discuss our results, for limiting cases, that may be appropriate for applications to space plasma environments including probable mechanism of escaping $\mathrm{H}^{+}$and $\mathrm{O}^{-}$from the Martian atmosphere.
\end{abstract}

Keywords. Electromagnetics (plasmas)

\section{Introduction}

It is known that the number density of neutrals is more than that of ions in chromospheric and lower ionospheric plasmas and, therefore, one must not dismiss the role of neutral dynamics in the study of dynamics of such media, i.e. partially ionised plasmas (hereafter PIP). Many observed phenomena in space are the direct consequence of fluctuations in the magnetic field, which makes the situation more complex; i.e. it appreciates change in both electric and magnetic fields. Consequently, species are subjected to higher random motion which leads to an unstable charge distribution. Also, it is worthwhile to see the effect of neutral dynamics on a perturbed magnetic field. A recent publication (Andersson and Ergun, 2012) has attracted attention by investigating the role of neutral wind in changing the ion distribution of the Martian atmosphere in the presence of a crustal magnetic field. In fact even incorporation of neutral collision has strongly modified some of the earlier results and has produced some new, exciting phonemonena both in pristine and dusty plasma environments (Shukla, 2007, and references therein). Hence there is a natural motivation to look for the EM (electromagnetic) part of the problem with a broader aspect in PIP. We would like to mention from the outset that there exists some work pertaining to the role of neutral species (Huba, 1990; Shaikh et al., 1999; Christopher and Jeremy, 2004; Song and Vasyliunas, 2005), to the best of our awareness, in the investigation of EM mode in PIP. Huba (1990) has presented a two-fluids model for PIP which was restricted to the analysis of the Rayleigh-Taylor type mode with the exclusion of neutral compressibility. Shaikh et al. (1999) have analysed high-frequency EM modes, with the same model, to study the effect of neutral dynamics in PIP. The work of Christopher and Jeremy (2004) was an experimental study on the Alfvén wave based on the simple theoretical model without $\boldsymbol{\nabla} \times \boldsymbol{B}$ effect in the neutral momentum equation and hence the subsequent effect of this term does not appear in their results. Song and Vasyliunas (2005) have studied the effect of a neutral atmosphere on dynamic processes of the magnetosphere-ionosphere-thermosphere system using the 
single-fluid model. In addition, and concerning the second part of our work (Sect. 3.3), there are a few publications (Amagishi and Tanaka, 1993; De Pontieu and Haerendel, 1998; Pécseli and Engvold, 2000; Khodachenko et al., 2004; Shaikh, 2009; Song and Vasyliunas, 2011) pertaining to partially ionised solar plasma. Amagishi and Tanaka (1993) have reported the influence of neutral, in a magnetised PIP, on the dispersion relation of the Alfvén wave. De Pontieu and Haerendel (1998) have shown the damping of the Alfvén by neutral-ion collision in order to put forward the probable mechanism of high magnitude force that gives rise to spicules in the solar chromosphere. Pécseli and Engvold (2000) have explained the dominance of the force exerted by the Alfvén wave in order to understand the levitation of prominence plasma with the help of the incompressible single-fluid model for PIP. Khodachenko et al. (2004) have reported the damping of MHD (magnetohydrodynamic) waves in the solar plasmas with the single-fluid model. Shaikh (2009) has shown, by simulation, the damping of the Alfvén wave by neutral collision with the single-fluid model. A recent publication (Song and Vasyliunas, 2011) has investigated, in detail, the heating mechanism of the solar atmosphere by the strong damping of Alfvén waves, with the consideration of frictional dissipation by ion-neutral collisions together with Joule heating. The above mentioned papers (Amagishi and Tanaka, 1993; De Pontieu and Haerendel, 1998; Khodachenko et al., 2004; Pécseli and Engvold, 2000; Shaikh, 2009; Song and Vasyliunas, 2005, 2011) have not considered (i) a two-fluids model, (ii) sources of free energy such as inhomogeneities in plasma and neutral fluids, magnetic field gradient and relative streaming, (iii) compressibility of both plasma and neutral fluids, and (iv) neutral dynamics (non-zero of $\tilde{n}_{\mathrm{n}}$ and $T_{\mathrm{n}}$ ). By consideration of these effects in our study we have obtained new results; i.e. with the two-fluid model we can analyse results in an extreme regime of frequency, sources of free energy are manifested in the form of either damping or growth of modes while compressibility of neutral brings forth new results. In addition, the above published works are either pertaining to lower planetary or solar PIP to discuss such a particular issue; i.e. damping of the Alfvén wave (and its consequences) considering the limited aspects of the neutral's involvement in homogeneous, cold PIP. It ought to be mentioned that these effects/considerations, i.e. sources of free energy, compressibilities, etc., are indubitably present and could be pertinent to the adduced examples shown in Table 1 . Ergo, we conclude this section by noting that there is no well-focused work which includes the effects of all aspects of neutral dynamics, in the presence of free energy, in magnetised PIP. The presented work, by consideration of missing studies/effects in previous research works, has shown significant advancement to develop a consistent study of collective processes, by incorporating neutral dynamics, in inhomogeneous, magnetised PIP.
The manuscript presents two major studies; i.e. the new EM instabilities seeded by relative streaming in association with neutral compressibility for hot, inhomogeneous PIP, and the strong influence of neutral dynamics on Alfvén wave propagation in the cold, homogeneous PIP with feasible assumptions.

In Sect. 2, we discuss a mathematical model, geometry and equilibrium. Section 3 analyses our dispersion relations with physically feasible assumptions. Additionally, justifications of assumptions in various physical situations are cited in the form of a table. We conclude with a discussion and summary of the work in Sect. 4. The detailed algebra involved in the derivation of dispersion relations is provided in Appendices $\mathrm{A}$ and $\mathrm{B}$.

\section{Basic equations}

In this section, to make the paper self contained, we reproduce (Shaikh and Das, 2002) the two-fluid model and equilibrium conditions. We note that the model allows us to analyse the fast and slow processes. In addition it also allows coupling between non-mechanical (Alfvén) and mechanical (neutral acoustic) waves. Following are the set of governing equations for the two-fluid model:

$\partial_{t} n_{\alpha}+\nabla \cdot\left(n_{\alpha} \boldsymbol{V}_{\boldsymbol{\alpha}}\right)=0$,

where $n_{\alpha}=$ number density, $V_{\alpha}=$ velocity of fluid and $\alpha$ represents the charged species and neutral particles. It is known that for many practical problems/applications the difference of the source and loss terms is small enough to neglect. Momentum equations for charged species are

$$
\begin{aligned}
m_{\mathrm{e}} n_{\mathrm{e}} D_{\mathrm{t}} \boldsymbol{V}_{\mathrm{e}}= & -e n_{\mathrm{e}}\left(\boldsymbol{E}-\boldsymbol{V}_{\mathrm{e}} \times \boldsymbol{B}\right)+\nabla P_{\mathrm{e}}+m_{\mathrm{e}} n_{\mathrm{e}} \boldsymbol{g} \\
& -n_{\mathrm{e}} m_{\mathrm{e}}\left[v_{\mathrm{en}}\left(\boldsymbol{V}_{\mathrm{e}}-\boldsymbol{V}_{\mathrm{n}}\right)-v_{\mathrm{ei}}\left(\boldsymbol{V}_{\mathrm{e}}-\boldsymbol{V}_{\mathrm{i}}\right)\right], \\
m_{\mathrm{i}} n_{\mathrm{i}} D_{\mathrm{t}} \boldsymbol{V}_{\mathrm{i}}= & e n_{\mathrm{i}}\left(\boldsymbol{E}-\boldsymbol{V}_{\mathrm{i}} \times \boldsymbol{B}\right)+\nabla P_{\mathrm{i}}+m_{\mathrm{i}} n_{\mathrm{i}} \boldsymbol{g} \\
& -n_{\mathrm{i}} m_{\mathrm{i}}\left[v_{\mathrm{in}}\left(\boldsymbol{V}_{\mathrm{i}}-\boldsymbol{V}_{\mathrm{n}}\right)-v_{\mathrm{ie}}\left(\boldsymbol{V}_{\mathrm{i}}-\boldsymbol{V}_{\mathrm{e}}\right)\right] .
\end{aligned}
$$

The symbols used in Eqs. (2) and (3) have their usual meaning. Now adding the electron (inertialess) and ion momentum equations,

$$
\begin{aligned}
m_{\mathrm{i}} n_{\mathrm{i}} D_{\mathrm{t}} \boldsymbol{V}_{\mathrm{i}}= & -\nabla\left(\frac{B^{2}}{8 \pi}+P\right)-n \nu_{\mathrm{eff}}\left(\boldsymbol{V}_{\mathrm{i}}-\boldsymbol{V}_{\mathrm{n}}\right) \\
& +\frac{c m_{\mathrm{e}} \mathrm{e}_{\mathrm{en}}}{4 \pi e} \nabla \times \boldsymbol{B}+m_{\mathrm{i}} n_{\mathrm{i}} \boldsymbol{g},
\end{aligned}
$$

where $v_{\text {eff }}=m_{\mathrm{i}} v_{\text {in }}+m_{\mathrm{e}} v_{\mathrm{en}}, m_{\mathrm{e}} v_{\mathrm{ei}}=m_{\mathrm{i}} v_{\mathrm{ie}}, n_{\mathrm{i}}=n_{\mathrm{e}}=n$ and $P=P_{\mathrm{e}}+P_{\mathrm{i}}$. We note that the law of conservation of momentum, quasi-neutrality, vector identity and Maxwell's equations with definition of current density $J$ are used in the derivation of Eq. (4). In our model, for partially ionised plasma, the neutrals being a "reservoir of momenta" are considered as a separate fluid coupled to the plasma fluid by 
mechanical and electromagnetic energy transformation. The momentum equation for neutrals is

$D_{\mathrm{t}} \boldsymbol{V}_{\mathrm{n}}=-\frac{\nabla P_{\mathrm{n}}}{m_{\mathrm{n}} n_{\mathrm{n}}}-(v)\left(\boldsymbol{V}_{\mathrm{n}}-\boldsymbol{V}_{\mathrm{i}}\right)+\frac{\nu_{\mathrm{ne}} c(\boldsymbol{\nabla} \times \boldsymbol{B})}{4 \pi n_{\mathrm{n}} e}+\boldsymbol{g}$,

where $v=v_{\text {ni }}+v_{\text {ne }}$ and we have used Maxwell's equations with the definition of current density $J$. The magnetic field evolution, from the ion-momentum balance equation, is

$$
\begin{aligned}
& \partial_{\mathrm{t}} \boldsymbol{B}=\boldsymbol{\nabla} \times\left(\boldsymbol{V}_{\mathrm{i}} \times \boldsymbol{B}\right)-\frac{m_{\mathrm{i}} c}{e} \nabla \times \frac{d \boldsymbol{V}_{\mathrm{i}}}{d t} \\
& -\frac{m_{\mathrm{i}} c \nu_{\mathrm{in}}}{e} \nabla \times\left(\boldsymbol{V}_{\mathrm{i}}-\boldsymbol{V}_{\mathrm{n}}\right)+\eta_{\mathrm{ei}} \nabla^{2} \boldsymbol{B}+\frac{m_{\mathrm{i}} c}{e}(\boldsymbol{\nabla} \times \boldsymbol{g}) .
\end{aligned}
$$

The system of Eqs. (1), (4)-(6) is closed by isothermal approximation for the equations of state; i.e. $P=n_{\mathrm{e}} T_{\mathrm{e}}+n_{\mathrm{i}} T_{\mathrm{i}} \sim$ $n_{\mathrm{i}}\left(T_{\mathrm{e}}+T_{\mathrm{i}}\right)$ with quasi-neutrality and $P_{\mathrm{n}}=n_{\mathrm{n}} T_{\mathrm{n}}$.

We consider that the magnetic field $B=B_{0}(x) \hat{e}_{\mathrm{z}}$, the plasma and neutral density scale lengths are in the positive $\mathrm{x}$ direction, while gravity and the magnetic field gradient are in the negative $\mathrm{x}$ direction. We note that there are no zeroth order velocities of ion and neutral species along $\mathrm{x}$-direction. For stationary behavior of equilibrium, setting $\frac{\partial}{\partial_{t}}=0$ in governing equations (Eqs. 1, 4-6) we get equilibrium conditions, i.e. Eqs. (7)-(10).

The x-component of Eq. (4), without frictional force, gives

$V_{\mathrm{A}}^{2}\left[L_{\mathrm{B}}^{-1}+\beta L_{\mathrm{P}}^{-1}\right]=-g$,

where $L_{\mathrm{P}}^{-1}=\frac{\partial_{\mathrm{x}} n_{\mathrm{o}}(x)}{n_{\mathrm{io}}}, L_{\mathrm{B}}^{-1}=\frac{\partial_{\mathrm{x}} B_{\mathrm{o}}(x)}{B_{\mathrm{o}}}, V_{\mathrm{A}}^{2}=\frac{B_{\mathrm{o}}^{2}}{4 \pi n_{\mathrm{io}} m_{i}}, C_{\mathrm{s}}^{2}=$ $\frac{2 T}{m_{\mathrm{i}}}$ and $\beta=\frac{C_{\mathrm{s}}^{2}}{V_{\mathrm{A}}^{2}}$. In the absence of gravity and friction Eq. (4) becomes

$\partial_{\mathrm{x}}\left(\frac{B_{\mathrm{o}}^{2}}{8 \pi}+P_{\mathrm{o}}\right)=0$

i.e. $\frac{B_{0}^{2}}{8 \pi}+P=$ constant, and $\beta=-\frac{L_{\mathrm{P}}}{L_{\mathrm{B}}}$. The Venus ionopause provides an example of magnetohydrodynamics static balance $(g=0)$.

The y-component of Eq. (4) is

$V_{\text {ioy }}-V_{\text {noy }}=-\frac{v_{\mathrm{en}} m_{\mathrm{e}} c}{4 \pi e v_{\mathrm{eff}} n_{\mathrm{io}}} \partial_{\mathrm{x}} B_{\mathrm{o}}$.

Now, the y-component of Eq. (5) gives

$V_{\text {ioy }}-V_{\text {noy }}=-\frac{v_{\text {ne }} c}{4 \pi n_{\text {io }} e} \frac{\partial_{\mathrm{x}} B_{\mathrm{o}}}{\left(v_{\mathrm{ni}}+v_{\mathrm{ne}}\right)}$.

It is to be noted that Eqs. (9) and (10) must be consistent and as a result, we must have

$\frac{v_{\text {ne }}}{v_{\text {ne }}+v_{\text {ni }}}=\frac{m_{\mathrm{e}} v_{\mathrm{en}}}{m_{\mathrm{i}} v_{\mathrm{in}}+m_{\mathrm{e}} v_{\mathrm{en}}}$.

This can be verified by substituting for $\nu_{\text {ne }}=\frac{m_{\mathrm{e}}}{m_{\mathrm{i}}} \frac{n_{\mathrm{i}}}{n_{\mathrm{n}}} v_{\mathrm{en}}$ and $v_{\mathrm{ni}}=\frac{n_{\mathrm{i}}}{n_{\mathrm{n}}} v_{\text {in }}$ with $m_{\mathrm{i}}=m_{\mathrm{n}} \gg m_{\mathrm{e}}$, which is valid for F region.

\section{Dispersion relation}

In order to derive the general EM dispersion relation, we have written the perturbed form of magnetic field evolution (Eq. 6), continuity and momentum equations for both fluids (Eqs. 1, 4, 5). The general dispersion relation is derived as a determinant in Appendix A (Eq. A8). The cross product of determinant involves extremely intricate algebra, hence we have restricted our discussion to relevant physics by using appropriate assumptions (ordering). We have delineated major results from the general EM dispersion relation (Eq. A8) under various limiting cases.

\subsection{Hot PIP}

Here, we assumed (a) low frequency $\left(\omega \ll \Omega_{\mathrm{i}}, \omega_{\mathrm{A}}, \omega_{\mathrm{t}}\right)$, (b) tightly coupled $\left(\omega \ll v_{\text {ni }}\right)$, (c) weakly resistive PIP $\omega \gg k^{2} \eta$, (d) $\left|\omega\left(\omega+i v_{i n}\right)\right| \ll \omega_{\mathrm{t}}^{2}$, (e) frequent collision and weakly magnetised PIP $\omega_{\mathrm{A}} \ll \Omega_{\mathrm{i}} \ll \nu_{\text {ni }}$ and (f) $1 \ll$ $(k L)^{2} \ll \omega_{\mathrm{t}}^{2} /\left|\omega\left(\omega+i v_{\text {in }}\right)\right|$, to derive the dispersion relation for this case. The general EM dispersion relation, Eq. (A8), under the above ordering scheme, and retaining the terms up to the $\delta^{2}$ is reduced to real and imaginary frequencies as follows:

$\omega_{0}=k V_{\mathrm{n} 0 \mathrm{y}}-\frac{\left(\Omega_{\mathrm{i}}^{2} \omega_{\mathrm{t}}^{2} k \Delta V\right) \omega_{\mathrm{A}}^{2} v_{\mathrm{ni}} \nu_{\mathrm{in}} k L_{\mathrm{n}}}{\left\{\omega_{\mathrm{A}}^{2} \nu_{\mathrm{ne}} \nu_{\mathrm{in}}\left(k L_{\mathrm{n}}\right)^{2}\right\}^{2}+\left\{\omega_{\mathrm{A}}^{2} \nu_{\mathrm{ni}} v_{\mathrm{in}} k L_{\mathrm{n}}\right\}^{2}}$,

$\gamma=\frac{\beta_{\mathrm{n}}^{2}\left\{\frac{\Omega_{\mathrm{i}}}{v_{\mathrm{in}}}\right\}^{2}}{\left(k L_{\mathrm{n}}\right)^{2}\left(\frac{\nu_{\mathrm{ne}}}{v_{\mathrm{ni}}}\right)-\left(\frac{\nu_{\mathrm{ni}}}{v_{\mathrm{ne}}}\right)} k \Delta V$,

where we have used $\beta_{\mathrm{n}} \ll \frac{v_{\mathrm{in}} \nu_{\mathrm{ni}}}{\Omega_{\mathrm{i}}^{2}} k L_{\mathrm{n}}$ with $\beta_{\mathrm{n}}=\frac{\omega_{\mathrm{t}}^{2}}{\omega_{\mathrm{A}}^{2}}, \omega_{\mathrm{t}}=$ $k V_{\mathrm{nt}}, \omega_{\mathrm{A}}=k V_{\mathrm{A}}, \Delta V=V_{\text {ioy }}-V_{\text {noy }}=-\frac{V_{\mathrm{A}}^{2}}{\Omega_{\mathrm{e}}} \frac{v_{\mathrm{en}}}{v_{\text {in }}} \frac{1}{L_{\mathrm{B}}}$,

$V_{\mathrm{nt}}^{2}=\frac{T_{\mathrm{n}}}{m_{\mathrm{n}}}, \quad L_{\mathrm{n}}^{-1}=\frac{\partial_{\mathrm{x}} n_{\mathrm{no}}(x)}{n_{\mathrm{no}}}, \quad \Omega_{\mathrm{i}}=\frac{e B_{0}}{m_{\mathrm{i}} c}$.

We note that the growth of mode is purely supported by neutral dynamics in hot, inhomogeneous, magnetised PIPs. Here assumption (f) is giving an indication of the timescale involved, for given parameters, in various applications. Also, we note that the first part of assumption (e); i.e. $k \ll \frac{\Omega_{\mathrm{i}}}{V_{\mathrm{A}}}$, imposes restriction on wavelength of mode. For the justification of assumption (f), we calculate thermal velocity for a given mass and temperature of the Venus ionosphere, the $\mathrm{D}$ region of Earth's ionosphere, the interstellar medium (ISM) and the detached region of the diverter of tokamak, which are shown in Table 1.

We notice, in Table 1, that either low frequency (LF) or extremely LF (ELF) can be supported by neutral-density scale length that can exist in the adduced regions of PIP. Hence it is interesting to look for an applicability of our results in 


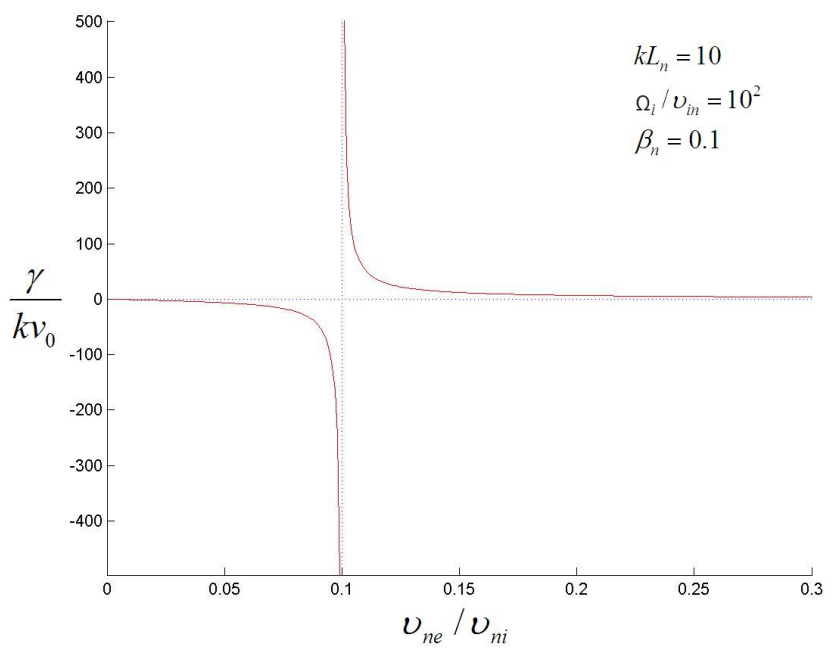

Fig. 1a. Plot based on Eq. (12) with an asymptote at 0.1 .

these regions. The above reported instability is on-set by relative streaming in association with neutral compressibility and collision timescale. When ions are magnetised, because of tight coupling, neutrals are also "magnetised" (i.e. following ions) and the field lines are loaded with more inertia. This will be manifested in the form of depletion of free energy and hence the growth rate of the above mode is reduced. Furthermore, due to mobility of neutrals the neutral pressure gradient flattens. Thus, collisions between charged and neutral particles, mass loading effect and the decrease in the pressure gradient slow down the relative streaming which has seeded this instability. Figure 1a shows the cutoff nature of the rationalised growth rate as a function of the ratio of collision frequencies of neutral with charged species. Asymptotes, seen in Fig. 1b, are independent of the ratio of frequencies as well as the height of the ionosphere. The probable avenue of understanding discontinuity lies in the mechanism of the interaction between magnetised ions and "magnetised" neutrals. It is natural to believe that there must be some finite time $\left(v_{\mathrm{ni}}^{-1}\right)$ lapses during the transfer of momentum/energy from magnetised ions to neutrals. During this time the medium has sufficient free energy in the form of net relative streaming to give rise to growth. However, upon elapsing this time period the neutrals are following ions and the relative streaming slows down. This reported new instability could be seen in the lower (equatorial) ionosphere and the solar chromosphere where neutrals are playing a dominant part in the dynamics of the media. Furthermore, analysing Eq. (12) leads to the disappearance of discontinuity; i.e. the denominator of Eq. (12) reduces to $-\left(k L_{\mathrm{n}}\right)^{2}\left(\frac{v_{\mathrm{ne}}}{v_{\mathrm{ni}}}\right)$, where we have used $v_{\mathrm{ne}}=\frac{m_{\mathrm{e}}}{m_{\mathrm{i}}} \frac{n_{\mathrm{i}}}{n_{\mathrm{n}}} v_{\mathrm{en}}, v_{\mathrm{ni}}=$ $\frac{n_{\mathrm{i}}}{n_{\mathrm{n}}} v_{\text {in }}$ and $m_{\mathrm{e}} v_{\mathrm{en}} \gg m_{\mathrm{i}} \nu_{\mathrm{in}}$. This is consistent with Fig. 1a.
Table 1. Justification of assumption (f) of Sect. 3.1.

\begin{tabular}{llll}
\hline Neutral in & $T(\mathrm{eV})$ & $V\left(\mathrm{~cm} \mathrm{~s}^{-1}\right)$ & Frequency \\
\hline Venus iono. $\left(\mathrm{CO}_{2}\right)$ & $.043-9.086$ & $3-6 \times 10^{4}$ & $\ll 1 \mathrm{~Hz}$ \\
D-Rgn $\left(\mathrm{NO}, \mathrm{O}_{2}\right)$ & .0172 & $2.3 \times 10^{4}$ & $\ll 0.1 \mathrm{~Hz}$ \\
ISM $\left(\mathrm{H}_{2}\right)$ & 0.862 & $6.3 \times 10^{5}$ & ELF \\
Tokamak $\left(\mathrm{H}_{2}\right)$ & 1 & $7.0 \times 10^{4}$ & ELF \\
\hline
\end{tabular}

\subsection{Cold PIP}

For cold PIP and no relative streaming, with same set of assumptions mentioned as in Sect. 3.1, we obtain the dispersion relation from Eq. (A8) of Appendix A:

$\omega_{\mathrm{c}}^{2}\left(X_{1}+i Y\right)+\omega_{\mathrm{c}}\left(\frac{k g}{\Omega_{\mathrm{i}}}\right)+k g(k L)^{-1}\left(X_{2}+i Y\right)=0$.

Where

$$
\begin{aligned}
& X_{1}=-1+2\left(k L_{p} k L_{\mathrm{B}}\right)^{-1}, \quad X_{2}=\frac{v_{\mathrm{ne}}^{2} \bar{\epsilon} \epsilon}{\Omega_{\mathrm{i}}^{2}}-1, \quad Y=\frac{v_{\mathrm{ne}} \bar{\epsilon}}{\Omega_{\mathrm{i}} k L_{\mathrm{B}}}, \\
& (k L)^{-1}=\left(k L_{\mathrm{p}}\right)^{-1}-\left(k L_{\mathrm{B}}\right)^{-1}, \bar{\epsilon}=1-\epsilon, \epsilon=\frac{v_{\mathrm{in}}}{v_{\mathrm{ni}}} .
\end{aligned}
$$

The above dispersion Eq. (13) is consistent with classical and collisional Rayleigh-Taylor. In order to get more meaningful results we can further delineate our result in categories with neutral dynamics $(\bar{\epsilon}=0)$ and without it $(\epsilon=0)$. However in the later case the neutrals, via the $\boldsymbol{\nabla} \times \boldsymbol{B}$ term, in the neutral momentum equation, have an important role to play. This can be seen by the presence of $\nu_{\text {ne }}$.

\subsection{Cold, homogeneous PIP}

In this section we derive the dispersion relation (Appendix B, Eqs. B6 and B7) for cold, homogeneous magnetic fields and number densities, without relative streaming.

$\left(\begin{array}{cc}D_{\mathrm{xx}} & D_{\mathrm{xy}} \\ D_{\mathrm{yx}} & D_{\mathrm{yy}}\end{array}\right)\left(\begin{array}{c}\tilde{v}_{\mathrm{x}} \\ \tilde{v}_{\mathrm{y}}\end{array}\right)=0$

where we have introduced the following notations with the assumption of strong coupling between ions and neutrals

$$
\begin{aligned}
& D_{\mathrm{xx}}=\frac{-i \omega}{\Omega_{\mathrm{i}}^{*}}, \quad D_{\mathrm{xy}}=\frac{\omega \omega_{\mathrm{B}}}{k^{2} V_{\mathrm{A}}^{* 2}}-1, \\
& D_{\mathrm{yx}}=\frac{-i \omega \omega_{\mathrm{B}}}{k^{2} V_{\mathrm{A}}^{* 2}}-\frac{i \nu_{\mathrm{n}} \omega}{\Omega_{\mathrm{i}}^{* 2}}, \quad D_{\mathrm{yy}}=-\frac{\nu_{\mathrm{ne}}}{\Omega_{\mathrm{i}}^{*}} .
\end{aligned}
$$

The above relation supports the important mode:

$k^{2}=\frac{\omega^{2}}{\tilde{V}_{\mathrm{A}}^{2}}\left[1+i\left(\frac{\eta \omega}{\hat{V}_{\mathrm{A}}^{2}}\right)\right]$.

Where

$$
\tilde{V}_{\mathrm{A}}^{2}=\dot{V}_{\mathrm{A}}^{2}\left[1+\left(\frac{\eta \omega}{\hat{V}_{\mathrm{A}}^{2}}\right)^{2}\right], \quad \dot{V}_{\mathrm{A}}^{2}=V_{\mathrm{A}}^{* 2}\left(1+\frac{\omega \nu_{\mathrm{ne}}}{\Omega_{\mathrm{i}}^{* 2}}\right)
$$



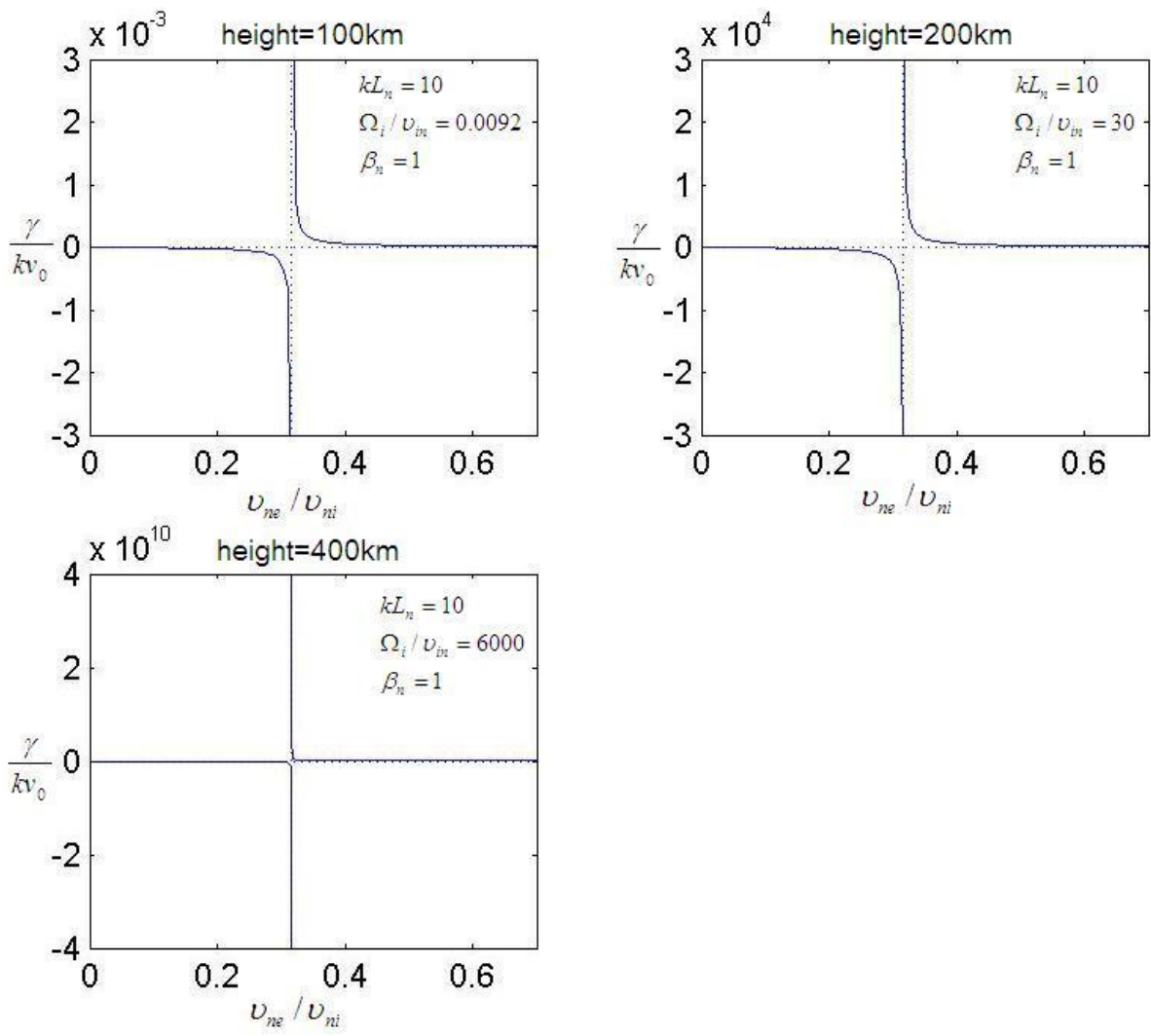

Fig. 1b. Plots based on Eq. (12) for different ionospheric ratios of gyrofrequency and neutral-ion collision frequency for given altitude of ionosphere.

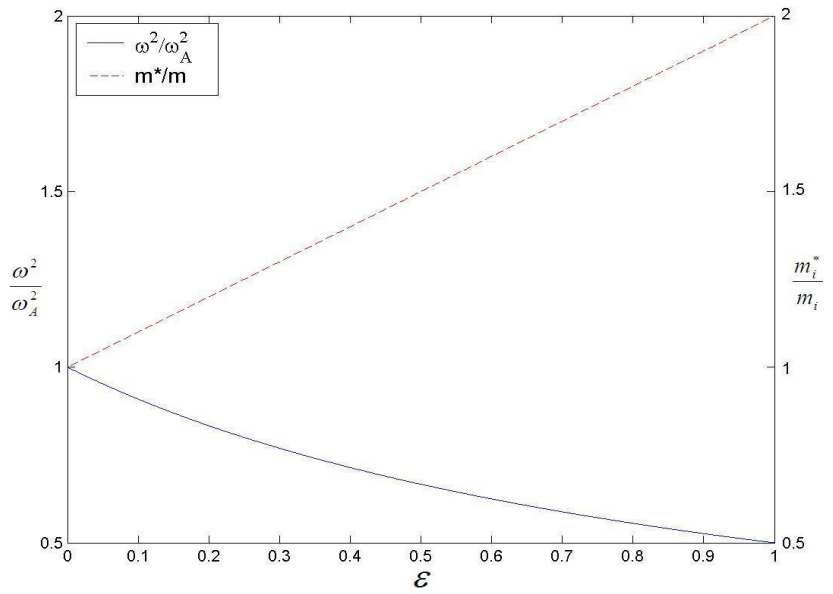

Fig. 2. Influence of neutral dynamics on Alfvén waves based on Eq. (15) for $V_{\text {ne }}$ tending to zero, non-resistive medium and strong coupling. Also, add-on mass effect $\left(m *_{\mathrm{i}}\right)$ on field line, due to strong coupling, is shown by dashed line.

$\Omega_{\mathrm{i}}^{*}=\frac{e B_{\mathrm{o}}}{m_{\mathrm{i}}^{*} c}, \quad V_{\mathrm{A}}^{* 2}=\frac{B_{\mathrm{o}}^{2}}{4 \pi n_{\mathrm{io}} m_{\mathrm{i}}^{*}}, \quad m_{\mathrm{i}}^{*}=m_{\mathrm{i}}\left(1+\frac{\frac{v_{\mathrm{in}}}{v_{\mathrm{ni}}}}{1-\frac{i \omega}{v_{\mathrm{ni}}}}\right)$.
From the above analytical result, Eq. (15), it is evident how modified inertia, electron-neutral collision and dissipative effect (diffusion velocity $\eta \omega$ ), due to neutral dynamics, are affecting Alfvén wave and cyclotron frequency. It is worth noting that the coefficient of ion mass in $m_{\mathrm{i}}^{*}$ is complex. This correction is reduced to $1+\epsilon$ or 1 for tight $\left(\omega \ll v_{\text {ni }}\right)$ and loose coupling $\left(\omega \gg v_{\mathrm{ni}}\right)$, respectively. Because of the strong coupling between ions and neutrals, they are like a body and their masses can be considered as the mass of a system. This is manifested as an additional mass in the Alfvén, cyclotron frequency and thermal velocity in our investigation. Figure 2 shows the influence of neutral dynamics on field line (mass loading) and subsequent effect on Alfvén wave velocity. It shows the effect of the ratio $\epsilon\left(=v_{\mathrm{in}} / \nu_{\mathrm{ni}}=m_{\mathrm{n}} n_{\mathrm{n} 0} / m_{\mathrm{i}} n_{\mathrm{i} 0}\right)$ on mass loading (dash line) on the field line and the Alfvén wave velocity (solid line). At $\epsilon=1$, i.e. maximum involvement of neutral dynamic, mass loading increases by $100 \%$ while Alfvén wave velocity decreases by $50 \%$ (for given $k$ ). We can conclude that the lower the ionisation rate, the higher the damping of the Alfvén wave. These results help to understand the transfer of energy from Alfvén wave to chromospheric PIP. Equation (15) can be analysed, for the strong coupling case, to obtain the propagation properties of a complex, inertia-loaded Alfvén wave in dissipative PIP by 
substituting complex $k$,

$2 k_{\mathrm{r}}^{2}=\left(\frac{\omega}{\tilde{V}_{\mathrm{A}}}\right)^{2} \pm\left(\frac{\omega}{\tilde{V}_{\mathrm{A}}}\right)^{2}\left[1+\left(\frac{\eta \omega}{\hat{V}_{\mathrm{A}}^{2}}\right)^{2}\right]^{\frac{1}{2}}$,

$2 k_{\mathrm{i}}^{2}=-\left(\frac{\omega}{\tilde{V}_{\mathrm{A}}}\right)^{2} \pm\left(\frac{\omega}{\tilde{V}_{\mathrm{A}}}\right)^{2}\left[1+\left(\frac{\eta \omega}{\dot{V}_{\mathrm{A}}^{2}}\right)^{2}\right]^{\frac{1}{2}}$.

Where

$\tilde{V}_{\mathrm{A}}^{2}=\frac{\left(\frac{B_{0}^{2}}{4 \pi}\right)}{n_{\mathrm{io}} \tilde{m}_{\mathrm{i}}}$,

$\tilde{m}_{\mathrm{i}}=m_{\mathrm{i}}\left[\frac{\left(1+\frac{\frac{v_{\mathrm{in}}}{v_{\mathrm{in}}}}{1-\frac{i \omega}{v_{\mathrm{ni}}}}\right)}{\left(1+\frac{\omega v_{\text {ne }}}{\Omega_{\mathrm{i}}^{* 2}}\right)\left(1+\left(\frac{\eta \omega}{\hat{V}_{\mathrm{A}}^{2}}\right)^{2}\right)}\right]$.

This is the novel expression of the modified Alfvén velocity $\left(\tilde{V}_{\mathrm{A}}^{2}\right)$ which includes the effect of the $\nabla \times \boldsymbol{B}$ term from the neutral momentum equation with electron-neutral collision, modified cyclotron frequency and the resistive nature of the medium. We also note that this expression is frequency dependent. Several comments are worth noting about the factor present $\left(m_{\mathrm{i}}\right)$ : (1) it is frequency dependent; (2) for loose coupling (high-frequency mode) there is no additional mass effect; (3) even in a strong coupling case, for an ideal MHD, the factor quantity in the square bracket could decrease under condition $\left(\frac{\omega v_{\mathrm{ne}}}{\Omega_{\mathrm{i}}^{2}}\right) \gg \epsilon(1+\epsilon)^{2}$ and, therefore, the mass loading effect will be replaced by decrease in mass. Physically this condition is feasible, for the given sensible ratio $\frac{\nu_{\text {ne }}^{2}}{\Omega_{\mathrm{i}}^{2}}$, under a high-frequency regime. Thus it can be stated that at high frequency, mass loading effect is replaced by unloading; i.e. mass decrease by a factor. Thus, in the high frequency regime Alfvén waves are less likely to survive. Hence, it can be concluded that our work appreciates the role of electronneutral collision which is absent in the previous publication (Pécseli and Engvold, 2000). We note that the positive sign, in Eqs. (16) and (17), is consistent with the physics involved; i.e. for absences of neutral dynamics it reduces them to classical Alfvén waves. It ought to be noted that (i) the ratio $\frac{k_{\mathrm{i}}}{k_{\mathrm{r}}}$ gives damping rate; (ii) $\frac{1}{k_{\mathrm{i}}}$ gives Alfvén damping scale $\left(H_{\mathrm{A}}\right)$ and (iii) the ratio $\frac{\omega^{2}}{k_{\mathrm{r}}^{2}}$ gives the phase velocity. It can be seen that above three properties of Alfvén wave propagation, by following simple algebra, are modified (Song and Vasyliunas, 2005). In addition these results are in consistent with the published work of lower planetary ionosphere (Christopher and Jeremy, 2004; Song and Vasyliunas, 2005, 2011).
Also dispersion Eq. (15) allows us to retrieve the relevant results of published work in solar PIP (Pécseli and Engvold, 2000; De Pontieu and Haerendel, 1998). Here it is clearly seen that neutral dynamics modifies both the restoring force (magnetic tension) and inertia in Alfvén oscillation. Hence, $B_{0}$ must be replaced by $B_{0} \exp \left[k_{\mathrm{i}} r \pm \frac{z}{H_{\mathrm{A}}}\right]$ in defining Alfvén waves to show their damping due to neutral dynamics in homogeneous, magnetised PIP. It shows that magnetic tension on field line decays exponentially in PIP. It is interesting to note that in plane electromagnetic-wave analysis $\tilde{V}_{\mathrm{A}}^{2}$ is replaced by $c^{2}$.

Furthermore, for an ideal MHD and tightly coupled case, Eq. (15) leads to a new result as:

$\omega_{0}^{2}= \pm \frac{\omega_{A}^{2}}{\epsilon_{1}} \mp \frac{1}{4}\left[k^{2} D_{\mathrm{A}} \bar{\epsilon}\right]^{2}$,

$\gamma=-\frac{k^{2} D_{\mathrm{A}} \bar{\epsilon}}{2}$

where we have introduced the new coefficient $D_{\mathrm{A}}=\frac{V_{\mathrm{A}}^{2} \nu_{\text {ne }}}{\Omega_{i}^{2}}$, and parameters $\epsilon_{1}=1+\epsilon$ and $\bar{\epsilon}=1-\epsilon$. We note that these coefficients and parameters are associated with neutral dynamics. These are new low-frequency EM modes supported by active participation of neutral in collective processes in PIP. The form of the mode, Eq. (18), is similar to that documented in the literature (Alexandrov et al., 1984) such as Langmuir, ES (electrostatic) ion cyclotron, ordinary waves. However the role of various terms are replaced by new terms; e.g. plasma and thermal frequencies both are replaced by Alfvén and collision frequencies. The close look at Eq. (18) has revealed that it is the quartic equation in $k$ which leads to complex wave vector $\boldsymbol{k}$. Therefore, in our case we do not expect a similar type of dispersion curve, as shown in literature of basic plasma physics (Alexandrov et al., 1984). We note that for $\epsilon=0$ in above relations gives a loosely coupled case.

\section{Discussion and conclusions}

We list following principal outcomes of our work:

1. In Sect. 3.1 we have reported a new growing mode with Doppler velocity for hot PIP which has no counterpart in fully ionised plasma (FIP). It is shown, numerically, that assumptions are accord well with space and laboratory plasma environments (see Table 1).

2. In Sect. 3.2 we have shown that cold PIP supports new modes similar to RT mode under the influence of neutral dynamics. In addition we have further delineated two new modes for homogeneous PIP in two limiting cases, i.e. for strong and loose coupling. 
3. In cold PIP, Sect. 3.3, we found that Alfvén waves are strongly influenced by neutral dynamics. We have shown that the factor (square bracket quantity) in the expression of modified mass, $\tilde{m}_{i}$, incorporates various dynamical quantities, and has overcome the restoring magnetic force/pressure. This effect is manifested as a reduction of amplitude of Alfvén oscillations. Hence we must revisit its long-scale coherence (few $\mathrm{Hz}$ ); i.e. its role of coupling is being restricted by, indirectly, the neutral dynamics. It is worth to mention that the influence of neutral dynamics is insignificant for highfrequency approximation.

4. We have presented a complex form of Alfvén wave velocity in PIP. Its propagation properties are discussed. Interestingly the mass loading effect, due to neutral dynamics, is also seen in cyclotron frequency and thermal velocity, i.e. replaced by $\Omega$ to $\Omega^{*}$ and $V_{\text {th }}$ to $V_{\text {th }}^{*}$. This may alter the overall dynamics of PIP.

5. Here the modified mass effect changes cyclotron frequency as well, i.e. $\Omega$ to $\Omega^{*}$. Hence, the ratio appears in the Whistler wave mode (in PIP) and should be $\frac{\omega}{\Omega^{*}}$ rather than $\frac{\omega}{\Omega}$. This implies that the Whistler wave mode is not transforming to low-frequency (MHD) mode while propagating from the magnetosphere to the ionosphere, which contradicts Song and Vasyliunas (2005). We offer, in the light of our work, the following physical explanation to resolve this contradiction: as the Whistler wave mode propagates from the magnetosphere (less collision) to the ionosphere (high-collision region) it "feels" the strong magnetic field (almost two orders higher) of the ionosphere, comparatively, that increases gyro frequency. However this effect is "ironed out" by the enhanced inertial effect which appeared in the modified $\Omega^{*}$; i.e. for $\epsilon=1$, mass increased by the factor of two. Thus, during propagation from the magnetosphere to the ionosphere the ratio $\frac{\omega}{\Omega^{*}}$ retains its magnitude, qualitatively, in the ionosphere as it was in the magnetosphere. Hence we conclude that ion cyclotron frequency is affected not only by magnetic field strength but neutral dynamics as well. This conclusion supports the propagation of the Whistler wave mode from the Earth's magnetosphere to the ionosphere without loosing its identity.

Finally, we wish to clarify the essential differences between the results presented in Sect. 3.3 and recent works (De Pontieu and Haerendel, 1998; Pécseli and Engvold, 2000; Khodachenko et al., 2004; Song and Vasyliunas, 2005; Shaikh, 2009; Song and Vasyliunas, 2011). The overall difference of our work from others consists in the chief objective of this paper: to bring forth the broader understanding of EM modes, stressing the role of neutral dynamics in collective processes with physically feasible assumptions rather than addressing particular issues. The expression of Alfvén ve- locity used by De Pontieu and Haerendel (1998) used simple mass-loaded Alfvén velocity. However, we have shown that by considering neutral dynamics, a few more effects (apart from mass loading factor) can be seen in our expression of Alfvén wave $\left(\tilde{V}_{\mathrm{A}}^{2}\right)$ velocity, e.g. $v_{\text {ne }}$ and magnetic diffusion velocity $\eta \omega$. The work of Pécseli and Engvold (2000) has not taken into account the compressibility of plasma and neutral fluid. Khodachenko et al. (2004) have used a single-fluid model which can appreciate the inertial effect of all species in the expression of Alfvén velocity. Besides this, their work was focused on viscous and Joule heating effects. Our mathematical model, considering various effects and geometry, is different compared to Song and Vasyliunas $(2005,2011)$. In addition, we have shown that plasma and neutral fluids are interacting via both mechanical and electromagnetic forces. This is apparent from the presence of the term $\boldsymbol{\nabla} \times \boldsymbol{B}$ in the neutral momentum equation. We note that the expressions of $k_{\mathrm{i}}$ and $k_{\mathrm{r}}$ which lead to a modified attenuation rate $\left(\frac{k_{\mathrm{i}}}{k_{\mathrm{r}}}\right)$ compared to that of Song and Vasyliunas (2005). We believe that, in the light of our work, the investigation of coupling solar wind-magnetosphere-ionosphere (Song and Vasyliunas, 2005) would be more appropriate with the consideration of inhomogeneities/sources of free energies, neutral density perturbation, its compressibility and the collision between ions and neutrals (friction term in neutral momentum equation). Because of strong coupling relative speed is reduced and hence interaction between ion and neutral (and vice versa) via charge exchange becomes significantly low. Hence we note that neutral dynamics is more dominant candidate for damping of Alfvén waves rather than the chargeexchange mechanism. Thus absence of a charge exchange process and different model in our work are major differences, apart from numerical analysis, compared to the recent publication (Shaikh, 2009).

Our most general electromagnetic dispersion relation with the inclusion of non-uniformities in number densities of ion, neutral and magnetic field has a wide scope of application. We have built-in resistivity and neutral dynamics in the model and therefore their comparative effect on Alfvén wave propagation can be seen at a glance. We have excluded collision frequencies between same species and electron-ions in our model. Their former exclusion could be justified by the fact that $v_{\text {ee }}$ and $v_{\text {ii }}$ are not contributing in transverse diffusion (being same mass and charge total momentum was conserved in the collision process). The isothermal equation of state approximation has been extensively used and has not missed out any major physical process so far. It ought to be stated that our work is indicative enough that the modified transport coefficient would have produced more dissipation effect in the system. Thus we conclude that inclusion of neutral dynamics has shown significant advancement in our understanding of EM modes in hot/cold, inhomogeneous/homogeneous, magnetised PIP. The algebraic expression of the perturbed magnetic field (Eq. A7) with the inclusion of neutral dynamics bring forth new physical effects. 
This expression can be used to reckon the time evolution of magnetic field in magnetised, inhomogeneous PIP. As far as concern to its application purpose a suitable local geometry is of paramount importance and it might be included in our future work. Nevertheless our geometry is suitable for the ionospheric region. Finally, we remark in the context of a recent publication (Andersson and Ergun, 2012) that our work (Sect. 3.3) may help to find one of the causes of fleeing Martian ions with neutral solar wind. The modified Alfvén wave velocity may provide enough power (local energy source) to Martian ions of $\mathrm{H}^{+}$and $\mathrm{O}^{-}$to escape. This might solve the problem of a low-powered solar wind condition which cannot pump enough energy in system. In addition, having a consistent chemistry of Mars' surface with the planet, once with water, the absence of other minerals (which can be formed only in the presence of water) on its surface can be justified.

\section{Appendix A}

We assume that all perturbed quantities are varying as $i(k y-\omega t)$. The linearised continuity equation for neutrals and ions are

$$
\frac{\tilde{n}_{\mathrm{n}}}{n_{\mathrm{no}}}=-i\left(\frac{L_{n}^{-1}}{\omega_{\mathrm{cn}}}\right) \tilde{V}_{\mathrm{nx}}+\left(\frac{k}{\omega_{\mathrm{cn}}}\right) \tilde{V}_{\mathrm{ny}},
$$

where $\omega_{\mathrm{cn}}=\omega-k V_{\mathrm{noy}}, L_{\mathrm{n}}^{-1}=\frac{1}{n_{\mathrm{no}}} \frac{\partial n_{\mathrm{no}}}{\partial x}$.

$$
\frac{\tilde{n}_{\mathrm{i}}}{n_{\mathrm{io}}}=-i\left(\frac{L_{\mathrm{p}}^{-1}}{\omega_{\mathrm{c}}}\right) \tilde{V}_{\mathrm{ix}}+\left(\frac{k}{\omega_{\mathrm{c}}}\right) \tilde{V}_{\mathrm{iy}},
$$

where $\omega_{\mathrm{c}}=\omega-k V_{\text {ioy }}, L_{\mathrm{p}}^{-1}=\frac{1}{n_{\mathrm{io}}} \frac{\partial n_{\mathrm{io}}}{\partial x}$.

\section{A1 Neutral momentum transfer equation}

$$
\begin{aligned}
D_{\mathrm{t}} V_{\mathrm{n}} & =-v_{\mathrm{ni}}\left(V_{\mathrm{n}}-V_{\mathrm{i}}\right)-v_{\mathrm{ne}}\left(V_{\mathrm{n}}-V_{\mathrm{e}}\right)-\frac{\nabla P_{\mathrm{n}}}{n_{\mathrm{n}} m_{\mathrm{n}}}+g \\
& =-v_{\mathrm{ni}}\left(V_{\mathrm{n}}-V_{\mathrm{i}}\right)-v_{\mathrm{ne}}\left[V_{\mathrm{n}}-V_{\mathrm{i}}+\frac{J}{n_{\mathrm{i}} e}\right]-\frac{\nabla P_{\mathrm{n}}}{n_{\mathrm{n}} m_{\mathrm{n}}}+g \\
D_{\mathrm{t}} V_{\mathrm{n}} & =-\left(V_{\mathrm{n}}-V_{\mathrm{i}}\right) v-\frac{v_{\mathrm{nn}} J}{n_{\mathrm{ie}}}-\frac{\nabla P_{\mathrm{n}}}{n_{\mathrm{n}} m_{\mathrm{n}}}+g \\
& =-\left(V_{\mathrm{n}}-V_{\mathrm{i}}\right) v-\frac{\nu_{\mathrm{ne}} c}{4 \pi n_{\mathrm{i}} e} \nabla \wedge B-\frac{\nabla P_{\mathrm{n}}}{n_{\mathrm{n}} m_{\mathrm{n}}}+g,
\end{aligned}
$$

where we have used $\nabla \times \boldsymbol{B}=\frac{4 \pi}{c} J, J=n_{\mathrm{i}} e\left(V_{\mathrm{i}}-V_{\mathrm{e}}\right)$ and $v=v_{\text {ni }}+v_{\text {ne. }}$. It is clear that the introduction of the magnetic field in the neutral momentum is due to the frictional forces of neutral with either species.

\section{A1.1 X-component of neutral momentum equation}

$$
\begin{aligned}
& \frac{\partial V_{\mathrm{n}}}{\partial t}+\left(V_{\mathrm{n}} \cdot \nabla V_{\mathrm{n}}\right)_{\mathrm{x}}=-\left(V_{\mathrm{n}}-V_{\mathrm{i}}\right)_{\mathrm{x}}\left(\nu_{\mathrm{ni}}+v_{\mathrm{ne}}\right) \\
& -\frac{\nu_{\mathrm{ne}} c(\nabla \times \boldsymbol{B})_{\mathrm{x}}}{4 \pi n_{\mathrm{i}} e}-\frac{\nabla_{\mathrm{x}} P_{\mathrm{n}}}{m_{\mathrm{n}} n_{\mathrm{n}}}-g,
\end{aligned}
$$

$$
\begin{aligned}
& -i \omega \tilde{V}_{\mathrm{nx}}+V_{\mathrm{noy}} i k \tilde{V}_{\mathrm{nx}}=-\left(v_{\mathrm{ni}}+v_{\mathrm{ne}}\right) \tilde{V}_{\mathrm{nx}}+\left(v_{\mathrm{ni}}+v_{\mathrm{ne}}\right) \tilde{V}_{\mathrm{ix}} \\
& -\frac{v_{\mathrm{ne}} c}{4 \pi n_{\mathrm{io}} e}\left(1-\frac{\tilde{n}_{\mathrm{i}}}{n_{\mathrm{io}}}\right)\left[\partial_{\mathrm{y}}\left(B_{\mathrm{oz}}+\tilde{B}_{\mathrm{z}}\right)\right]-\frac{\nabla_{\mathrm{x}} \tilde{P}_{\mathrm{n}}}{m_{\mathrm{n}} n_{\mathrm{n}}} \\
& -i \omega \tilde{V}_{\mathrm{nx}}+V_{\mathrm{noy}} i k \tilde{V}_{\mathrm{nx}}+\left(v_{\mathrm{ni}}+v_{\mathrm{ne}}\right) \tilde{V}_{\mathrm{nx}}=\left(v_{\mathrm{ni}}+v_{\mathrm{ne}}\right) \tilde{V}_{\mathrm{ix}} \\
& -\frac{v_{\mathrm{ne}} c}{4 \pi n_{\mathrm{io}} e} \partial_{\mathrm{y}} \tilde{B}_{\mathrm{z}}-v_{\mathrm{nt}}^{2} \frac{\nabla_{\mathrm{x}} n_{\mathrm{n} 0}\left(\frac{\tilde{n}_{\mathrm{n}}}{n_{\mathrm{n} 0}}\right),}{n_{\mathrm{no}}} \\
& -i \tilde{V}_{\mathrm{nx}}\left\{\omega_{\mathrm{cn}}+i\left(v_{\mathrm{ni}}+v_{\mathrm{ne}}\right)\right\}=\left(v_{\mathrm{ni}}+v_{\mathrm{ne}}\right) \tilde{V}_{\mathrm{ix}} \\
& -\frac{i k v_{\mathrm{ne}} c}{4 \pi n_{\mathrm{io}} e} \tilde{B}-v_{\mathrm{nt}}^{2} \frac{i L_{\mathrm{n}}^{-2}}{\omega_{\mathrm{cn}}} \tilde{V}_{\mathrm{nx}}+\frac{\omega_{\mathrm{t}}^{2}}{k L_{\mathrm{n}} \omega_{\mathrm{cn}}} \tilde{V}_{\mathrm{ny}}, \\
& -i\left[\omega_{\mathrm{cn}}+i\left(v_{\mathrm{ni}}+v_{\mathrm{ne}}\right)-\frac{\omega_{\mathrm{t}}^{2}}{k^{2} L_{\mathrm{n}}^{2}} \frac{1}{\omega_{\mathrm{cn}}}\right] \\
& \tilde{V}_{\mathrm{nx}}=\left(v_{\mathrm{ni}}+v_{\mathrm{ne}}\right) \\
& \tilde{V}_{\mathrm{ix}}-\frac{i k v_{\mathrm{ne}} c}{4 \pi n_{\mathrm{io}} e} \tilde{B}+\left(\frac{\omega_{\mathrm{t}}^{2}}{k L_{\mathrm{n}} \omega_{\mathrm{cn}}}\right) \tilde{V}_{\mathrm{ny}},
\end{aligned}
$$$$
\tilde{V}_{\mathrm{nx}}=\frac{i \nu_{\mathrm{ni}}}{\omega_{\mathrm{nx}}} \tilde{V}_{\mathrm{ix}}+\frac{k \nu_{\mathrm{ne}} c}{4 \pi n_{\mathrm{io}} e} \frac{\tilde{B}}{\omega_{\mathrm{nx}}} \hat{e}_{\mathrm{x}}+\left(\frac{i \omega_{\mathrm{t}}^{2}}{k L_{\mathrm{n}} \omega_{\mathrm{cn}}}\right) \frac{\tilde{V}_{\mathrm{ny}}}{\omega_{\mathrm{nx}}} \hat{e}_{\mathrm{x}},
$$

where $v_{\mathrm{ni}} \gg v_{\mathrm{ne}}$ and $\omega_{\mathrm{nx}}=\omega_{\mathrm{cn}}+i v_{\mathrm{ni}}-\frac{\omega_{\mathrm{t}}^{2}}{\left(k L_{\mathrm{n}}\right)^{2}} \frac{1}{\omega_{\mathrm{cn}}}$.

\section{A1.2 Y-component of neutral momentum equation}

$$
\begin{array}{r}
\partial_{\mathrm{t}} \tilde{V}_{\text {ny }}+V_{\text {noy }} \partial_{\mathrm{y}} \tilde{V}_{\text {ny }}=-v_{\text {ni }}\left(\tilde{V}_{\text {ny }}\right)+\tilde{V}_{\text {iy }} v_{n i}-\frac{v_{\text {ne }} c}{4 \pi n_{\text {io }} e} \\
\left(\frac{\tilde{n}_{\mathrm{i}}}{n_{\mathrm{io}}} \partial_{\mathrm{x}} B_{\mathrm{oz}} \hat{e}_{\mathrm{y}}\right)-\frac{\omega_{\mathrm{t}}^{2}}{k L_{\mathrm{n}} \omega_{\mathrm{cn}}} \tilde{V}_{\mathrm{nx}}-\frac{i \omega_{\mathrm{t}}^{2}}{\omega_{\mathrm{cn}}} \tilde{V}_{\mathrm{ny}},
\end{array}
$$

where we have assumed $v_{\text {ni }}>v_{\text {ne }}$.

$$
\begin{gathered}
-i\left(\omega_{\mathrm{cn}}+i \nu_{\mathrm{ni}}-\frac{\omega_{\mathrm{t}}^{2}}{\omega_{\mathrm{cn}}}\right) \tilde{V}_{\mathrm{ny}}=v_{\mathrm{ni}} \tilde{V}_{\mathrm{iy}}- \\
\frac{\nu_{\mathrm{ne}} c}{4 \pi n_{\mathrm{io}} e} \partial_{\mathrm{x}} B_{\mathrm{o}}\left(\frac{\tilde{n}_{i}}{n_{\mathrm{io}}}\right)-\frac{\omega_{\mathrm{t}}^{2}}{k L_{\mathrm{n}} \omega_{\mathrm{cn}}} \tilde{V}_{\mathrm{nx}}, \\
\tilde{V}_{\mathrm{ny}}=i \frac{v_{\mathrm{ni}}}{\omega_{\mathrm{ny}}} \tilde{V}_{\mathrm{iy}}-\frac{i v_{\mathrm{ne}} c}{4 \pi n_{\mathrm{io}} e} \frac{\partial_{\mathrm{x}} B_{\mathrm{o}}}{\omega_{\mathrm{ny}}}\left(\frac{\tilde{n}_{\mathrm{i}}}{n_{\mathrm{io}}}\right) \\
-i \frac{\omega_{\mathrm{t}}^{2}}{\omega_{\mathrm{ny}} k L_{\mathrm{n}} \omega_{\mathrm{cn}}} \tilde{V}_{\mathrm{nx}} .
\end{gathered}
$$

Where $\omega_{\mathrm{ny}}=\omega_{\mathrm{cn}}+i v_{\mathrm{ni}}-\frac{\omega_{\mathrm{t}}^{2}}{\omega_{\mathrm{cn}}}$.

Substituting Eq. (A2) in Eq. (A1) we get

$$
\begin{aligned}
\tilde{V}_{\mathrm{nx}} & =\left[\frac{i \nu_{\mathrm{ni}}}{\omega_{\mathrm{nx}} D_{\mathrm{n}}}-i \frac{f_{\mathrm{x}}}{\omega_{\mathrm{c}}} \frac{1}{k L_{\mathrm{p}}}\right] \tilde{V}_{\mathrm{ix}} \\
& -\left[\frac{\omega_{\mathrm{t}}^{2} \nu_{\mathrm{ni}}}{\omega_{\mathrm{nx}} \omega_{\mathrm{ny}} \omega_{\mathrm{cn}} D_{\mathrm{n}} k L_{\mathrm{n}}}+\frac{f_{\mathrm{x}}}{\omega_{\mathrm{c}}}\right] \tilde{V}_{\mathrm{iy}} \\
& +\frac{k v_{\mathrm{ne}} c}{4 \pi n_{\mathrm{io}} e} \frac{\tilde{B}}{\omega_{\mathrm{nx}} D_{\mathrm{n}}} \hat{e}_{\mathrm{x}},
\end{aligned}
$$


where $f_{\mathrm{x}}=k \Delta V v_{\mathrm{in}} \frac{k L_{\mathrm{n}}}{\omega_{\mathrm{t}}^{2}} \omega_{\mathrm{cn}}$.

Now substituting Eq. (A3) in Eq. (A2) so we have

$$
\begin{aligned}
\tilde{V}_{\mathrm{ny}} & =\left[\frac{\omega_{\mathrm{t}}^{2} v_{\mathrm{ni}}}{k L_{\mathrm{n}} \omega_{\mathrm{cn}} \omega_{\mathrm{nx}} \omega_{\mathrm{ny}} D_{\mathrm{n}}}-\frac{f_{\mathrm{y}}}{k L_{\mathrm{p}} \omega_{\mathrm{c}}}\right] \tilde{V}_{\mathrm{ix}} \\
+ & {\left[\frac{i \nu_{\mathrm{ni}}}{\omega_{\mathrm{ny}} D_{\mathrm{n}}}-i \frac{f_{\mathrm{y}}}{\omega_{\mathrm{c}}}\right] \tilde{V}_{\mathrm{iy}} } \\
& -\frac{i k c^{2} \omega_{\mathrm{t}}^{2} \Omega_{i} \nu_{\mathrm{ne}}}{k L_{\mathrm{n}} \omega_{\mathrm{cn}} \omega_{\mathrm{nx}} \omega_{\mathrm{ny}} \omega_{\mathrm{pi}}^{2} D_{\mathrm{n}}}\left(\frac{\tilde{B}}{B_{0}}\right),
\end{aligned}
$$

where

$$
f_{\mathrm{y}}=\frac{v_{\mathrm{in}} k \Delta V\left(k L_{\mathrm{n}}\right)^{2} \omega_{\mathrm{nx}} \omega_{\mathrm{cn}}^{2}}{\omega_{\mathrm{t}}^{4}}, D_{\mathrm{n}}=1-\frac{\omega_{\mathrm{t}}^{4}}{\left(k L_{\mathrm{n}}\right)^{2} \omega_{\mathrm{cn}}^{2} \omega_{\mathrm{nx}} \omega_{\mathrm{ny}}} .
$$

\section{A2 Plasma momentum equation}

\section{A2.1 X-component}

$$
\begin{aligned}
& \omega_{\mathrm{c}} \tilde{V}_{\mathrm{ix}}=-i g\left\{-i \frac{1}{\omega} \frac{1}{L_{\mathrm{p}}} \tilde{V}_{\mathrm{ix}}+\frac{k}{\omega} \tilde{V}_{\mathrm{iy}}\right\}-i v_{\mathrm{in}} \tilde{V}_{\mathrm{ix}} \\
& +\frac{i \nu_{\mathrm{in}} k v_{\mathrm{ne}} c}{4 \pi n_{\mathrm{io}} e \omega_{\mathrm{nx}}} \frac{B_{\mathrm{o}}}{D_{\mathrm{n}}}\left(\frac{\tilde{B}}{B_{\mathrm{o}}}\right) \hat{e}_{\mathrm{x}} \\
& +i v_{\mathrm{in}}\left\{\frac{i v_{\mathrm{ni}}}{\omega_{\mathrm{nx}} D_{\mathrm{n}}}-\frac{i f_{\mathrm{x}}}{\omega_{c} k L_{\mathrm{p}}}\right\} \tilde{V}_{\mathrm{ix}}+\left\{-\frac{i v_{\mathrm{in}} \omega_{t}^{2} \nu_{\mathrm{ni}}}{\omega_{\mathrm{nx}} \omega_{\mathrm{ny}} D_{\mathrm{n}} k L_{\mathrm{n}}} \frac{f_{\mathrm{x}}}{\omega_{\mathrm{c}}}\right\} \tilde{V}_{\mathrm{iy}}, \\
& \left\{-\omega_{\mathrm{c}}-\frac{k g}{\omega_{\mathrm{c}} k L_{\mathrm{p}}}-i v_{\mathrm{in}}-\frac{v_{\mathrm{in}} \nu_{\mathrm{ni}}}{\omega_{\mathrm{nx}} D_{\mathrm{n}}}+\frac{f_{\mathrm{x}}}{k L_{\mathrm{p}} \omega_{\mathrm{c}}} v_{\mathrm{in}}\right\} \tilde{V}_{\mathrm{ix}} \\
& +\left\{-\frac{i g k}{\omega_{\mathrm{c}}}-\frac{\omega_{\mathrm{t}}^{2} \nu_{\mathrm{ni}}}{\omega_{\mathrm{nx}} \omega_{\mathrm{ny}} D_{\mathrm{n}} k L_{\mathrm{n}}}+\frac{i f_{\mathrm{x}} \nu_{\mathrm{in}}}{\omega_{\mathrm{c}}}\right\} \tilde{V}_{\mathrm{iy}} \\
& +\frac{i v_{\mathrm{in}} k v_{\mathrm{ne}} c}{4 \pi n_{\mathrm{io}} e \omega_{\mathrm{nx}}} \frac{B_{\mathrm{o}}}{D_{\mathrm{n}}}\left(\frac{\tilde{B}}{B_{\mathrm{o}}}\right) \hat{e}_{\mathrm{x}}=0
\end{aligned}
$$

\section{A2.2 Y-component}

$$
\begin{aligned}
\omega_{\mathrm{c}} \tilde{V}_{\mathrm{iy}} & =k C_{\mathrm{s}}^{2}\left[-i \frac{L_{\mathrm{p}}^{-1}}{\omega_{\mathrm{c}}} \tilde{V}_{\mathrm{ix}}+\frac{k}{\omega_{c}} \tilde{V}_{\mathrm{iy}}\right]-i \nu_{\mathrm{in}} \tilde{V}_{i y} \\
& +i \nu_{\mathrm{in}}\left\{\frac{\omega_{\mathrm{t}}^{2} \nu_{\mathrm{ni}}}{k L_{\mathrm{n}} \omega_{\mathrm{cn}} \omega_{\mathrm{nx}} \omega_{\mathrm{ny}} D_{\mathrm{n}}} \tilde{V}_{\mathrm{ix}}-\frac{f_{\mathrm{y}}}{k L_{\mathrm{p}} \omega_{\mathrm{c}}} \tilde{V}_{\mathrm{ix}}\right. \\
& \left.+\frac{i \nu_{\mathrm{ni}}}{\omega_{\mathrm{ny}} D_{\mathrm{n}}} \tilde{V}_{\mathrm{iy}}+\frac{i f_{\mathrm{y}}}{\omega_{\mathrm{c}}} \tilde{V}_{\mathrm{iy}}\right\} \\
& +i \nu_{\mathrm{ni}}\left(\frac{i k c^{2} \omega_{\mathrm{t}}^{2} \Omega_{\mathrm{i}} \nu_{\mathrm{ne}}}{k L_{\mathrm{n}} \omega_{\mathrm{cn}} \omega_{\mathrm{nx}} \omega_{\mathrm{ny}} \omega_{\mathrm{pi}}^{2}}\right)\left(\frac{\tilde{B}}{B_{0}}\right)
\end{aligned}
$$

\section{A3 Magnetic field evolution}

$$
\begin{aligned}
\partial_{\mathrm{t}} \boldsymbol{B}= & \nabla \times\left(\boldsymbol{V}_{\mathrm{i}} \times \boldsymbol{B}\right)-\frac{m_{\mathrm{i}} c}{e} \nabla \times \frac{d \boldsymbol{V}_{\mathrm{i}}}{d t}-\frac{m_{\mathrm{i}} c}{e} v_{\mathrm{in}} \nabla \times\left(\boldsymbol{V}_{\mathbf{i}}-\boldsymbol{V}_{\mathbf{n}}\right) \\
& +\eta_{\mathrm{ei}} \nabla^{2} \boldsymbol{B}+\frac{m_{\mathrm{i}} c}{e}(\nabla \times \boldsymbol{g}) \\
-i \omega \tilde{B}_{\mathrm{z}}= & -V_{\mathrm{ioy}} \partial_{\mathrm{y}} \tilde{B}_{\mathrm{z}}-\left(\partial_{\mathrm{x}} B_{\mathrm{oz}}\right) \tilde{V}_{\mathrm{ix}}-B_{\mathrm{oz}} \partial_{\mathrm{y}} \tilde{V}_{\mathrm{iy}} \\
& +\frac{m_{\mathrm{i}} c}{e} \partial_{\mathrm{y}} \frac{d}{d t} \tilde{V}_{\mathrm{ix}}+\frac{m_{\mathrm{i}} c}{e} v_{\mathrm{in}} \partial_{\mathrm{y}}\left(\tilde{V}_{\mathrm{ix}}-\tilde{V}_{\mathrm{nx}}\right) \\
& +\eta_{\mathrm{ei}} \nabla^{2} \tilde{B}_{\mathrm{z}},
\end{aligned}
$$

where we have assumed that there is no velocity gradient (shear).

$$
\begin{aligned}
& \tilde{B}_{\mathrm{z}}\left(-i \omega+i k V_{\mathrm{ioy}}+\eta_{\mathrm{ei}} k_{\mathrm{y}}^{2}\right)=-\tilde{V}_{\mathrm{ix}} \partial_{\mathrm{x}} B_{\mathrm{oz}}-i k \tilde{V}_{\mathrm{iy}} B_{\mathrm{oz}} \\
& +\frac{m_{\mathrm{i}} c}{e} i k(-i \omega) \tilde{V}_{\mathrm{ix}}+i \frac{m_{\mathrm{i}} c}{e} v_{\mathrm{in}} k\left(\tilde{V}_{\mathrm{ix}}-\tilde{V}_{\mathrm{nx}}\right) \\
& \tilde{B}_{\mathrm{z}}\left[-i\left(\omega_{\mathrm{m}}\right)\right]= \\
& \begin{aligned}
\frac{m_{\mathrm{i}} c}{e} k\left(\omega-k V_{\mathrm{ioy}}+i \nu_{\mathrm{in}}\right) \tilde{V}_{\mathrm{ix}}-i \frac{m_{\mathrm{i}} c}{e} v_{\mathrm{in}} k \tilde{V}_{\mathrm{nx}}-i k y B_{\mathrm{oz}} \tilde{V}_{\mathrm{iy}}+
\end{aligned}
\end{aligned}
$$

Substituting Eq. (A3) we have

$$
\begin{aligned}
\frac{\tilde{B}_{\mathrm{z}}}{B_{\mathrm{o}}} & =\frac{1}{\omega_{\mathrm{B}}}\left\{-i L_{\mathrm{B}}^{-1}+\frac{i k}{\Omega_{\mathrm{i}}}\left(\omega_{\mathrm{c}}+i v_{\mathrm{in}}\right)-\frac{v_{\mathrm{in}}}{\Omega_{\mathrm{i}}} k\left[\frac{i v_{\mathrm{ni}}}{\omega_{\mathrm{nx}} D_{\mathrm{n}}}-\frac{f_{\mathrm{x}}}{\omega_{\mathrm{c}} k L_{\mathrm{p}}}\right]\right\} \tilde{V}_{\mathrm{ix}} \\
& +\frac{1}{\omega_{\mathrm{B}}}\left\{k-\frac{\nu_{\mathrm{in}}}{\Omega_{\mathrm{i}}} k\left[\frac{\omega_{\mathrm{t}}^{2}}{\omega_{\mathrm{nx}} \omega_{\mathrm{ny}} \omega_{\mathrm{cn}} D_{\mathrm{n}} k L_{\mathrm{n}}}+\frac{f_{\mathrm{x}}}{\omega_{\mathrm{c}}}\right]\right\} \tilde{V}_{\mathrm{iy}} \cdot(\mathrm{A} 7)
\end{aligned}
$$

Where

$$
\omega_{\mathrm{B}}=\omega_{\mathrm{c}}+i k_{\mathrm{y}}^{2} \eta_{\mathrm{ei}}-\frac{c^{2} k^{2} \nu_{\mathrm{in}} \nu_{\mathrm{ne}}}{\omega_{\mathrm{pe}}^{2} \omega_{\mathrm{nx}} D_{\mathrm{n}}}, \omega_{\mathrm{m}}=\omega_{\mathrm{c}}+i \nu_{\mathrm{in}}-\frac{\nu_{\mathrm{in}} \nu_{\mathrm{ni}}}{D_{\mathrm{n}} \omega_{\mathrm{nx}}} .
$$

Now Substituting Eq. (A7) into Eq. (A5) and Eq. (A6) we obtain the following general dispersion relation:

$$
\operatorname{Det}\left(\begin{array}{ll}
D_{\mathrm{xx}} & D_{\mathrm{xy}} \\
D_{\mathrm{yx}} & D_{\mathrm{yy}}
\end{array}\right)=0 \text {. }
$$

Where

$$
\begin{aligned}
D_{\mathrm{xx}}= & -\omega_{\mathrm{m}}+\frac{\omega_{\mathrm{A}}^{2}}{\omega_{\mathrm{B}} \Omega_{\mathrm{i}}} v_{\mathrm{ne}}\left[\left(k L_{\mathrm{B}}\right)^{-1}-\frac{\omega_{\mathrm{m}}}{\Omega_{\mathrm{i}}}\right] D_{\mathrm{x}}-\frac{k g}{\omega_{\mathrm{c}} k L_{\mathrm{p}}} \\
& -i v_{\mathrm{in}} \omega_{\mathrm{t}}^{2}\left[\frac{i \omega_{\mathrm{A}}^{2} \nu_{\mathrm{ne}}}{D_{\mathrm{n}} \Omega_{\mathrm{i}} \omega_{\mathrm{cn}} \omega_{\mathrm{nx}} \omega_{\mathrm{ny}} \omega_{\mathrm{c}} k L_{\mathrm{B}} k L_{\mathrm{n}} k L_{\mathrm{p}}}\right] \\
& -\frac{v_{\mathrm{ni}} v_{\mathrm{in}} \omega_{\mathrm{t}}^{2}}{k L_{\mathrm{n}} \omega_{\mathrm{cn}} \omega_{\mathrm{nx}} \omega_{\mathrm{ny}} D_{\mathrm{n}}} \\
& -\frac{\omega_{\mathrm{t}}^{2} v_{\mathrm{in}} \omega_{\mathrm{A}}^{2} v_{\mathrm{ne}}\left[\left(k L_{\mathrm{B}}\right)^{-1}-\frac{\omega_{\mathrm{m}}}{\Omega_{\mathrm{i}}}\right]}{D_{\mathrm{n}} \Omega_{\mathrm{i}} \omega_{\mathrm{B}} \omega_{\mathrm{cn}} \omega_{\mathrm{nx}} \omega_{\mathrm{ny}} k L_{\mathrm{n}}}
\end{aligned}
$$

$$
\begin{aligned}
D_{\mathrm{xy}}= & -i k L_{\mathrm{p}}^{-1} \omega_{\mathrm{m}}+\frac{i v_{\mathrm{ne}} v_{\mathrm{in}} \omega_{\mathrm{A}}^{2}}{D_{\mathrm{n}} \Omega_{\mathrm{i}} \omega_{\mathrm{ny}} k L_{\mathrm{B}} k L_{\mathrm{p}}}\left(\frac{1}{\omega_{\mathrm{c}}}-\frac{1}{\omega_{\mathrm{B}}}\right) \\
& +\frac{v_{\mathrm{ne}} \omega_{\mathrm{A}}^{2}}{\Omega_{\mathrm{e}} \omega_{\mathrm{B}}\left(k L_{\mathrm{B}}\right)^{2}}+\frac{i v_{\mathrm{ne}} \omega_{\mathrm{A}}^{2}}{\Omega_{\mathrm{i}} \omega_{\mathrm{B}}} D_{\mathrm{x}}-i \frac{k g}{\omega_{\mathrm{c}}} \\
& -\frac{i \nu_{\mathrm{in}} \omega_{\mathrm{t}}^{2}}{D_{\mathrm{n}} \omega_{\mathrm{cn}} \omega_{\mathrm{nx}} \omega_{\mathrm{ny}} k L_{\mathrm{n}}}\left[\nu_{\mathrm{ni}}-\frac{\nu_{\mathrm{ne}} \omega_{\mathrm{A}}^{2}}{\Omega_{\mathrm{i}} \omega_{\mathrm{c}} k L_{\mathrm{B}}}\right] \\
& -v_{\mathrm{in}} \omega_{\mathrm{t}}^{2}\left[\frac{-i v_{\mathrm{ne}} \omega_{\mathrm{A}}^{2}}{D_{\mathrm{n}} \Omega_{\mathrm{i}} \omega_{\mathrm{B}} \omega_{\mathrm{cn}} \omega_{\mathrm{nx}} \omega_{\mathrm{ny}} k L_{\mathrm{n}}}\right]
\end{aligned}
$$




$$
\begin{aligned}
D_{\mathrm{yx}}= & \frac{-i \omega_{\mathrm{A}}^{2}}{\omega_{\mathrm{B}}}\left[\left(k L_{\mathrm{B}}\right)^{-1}-\frac{\omega_{\mathrm{m}}}{\Omega_{\mathrm{i}}}\right] \\
& -\frac{i v_{\mathrm{ne}} v_{\mathrm{in}} \omega_{\mathrm{A}}^{2}}{D_{\mathrm{n}} \Omega_{\mathrm{i}} \omega_{\mathrm{c}} \omega_{\mathrm{ny}} k^{2} L_{\mathrm{p}} L_{\mathrm{B}}}+\frac{i v_{\mathrm{ne}} \omega_{\mathrm{A}}^{2}}{\Omega_{\mathrm{i}} \omega_{\mathrm{B}}\left(k L_{\mathrm{B}}\right)^{2}} D_{\mathrm{y}} \\
& -\frac{k \Delta V}{k L_{\mathrm{p}}} \frac{\nu_{\mathrm{in}}}{\omega_{\mathrm{c}}}-\frac{i\left(k C_{\mathrm{s}}\right)^{2}}{k L_{\mathrm{p}} \omega_{\mathrm{c}}} \\
& +i \frac{\nu_{\mathrm{ni}} \nu_{\mathrm{in}} \omega_{\mathrm{t}}^{2}}{\omega_{\mathrm{cn}} \omega_{\mathrm{nx}} \omega_{\mathrm{ny}} k L_{\mathrm{n}} D_{\mathrm{n}}} \\
& -\frac{i \nu_{\mathrm{in}} \omega_{\mathrm{t}}^{2} \nu_{\mathrm{ne}} \omega_{\mathrm{A}}^{2}}{\omega_{\mathrm{B}} \Omega_{\mathrm{i}} \omega_{\mathrm{cn}} \omega_{\mathrm{nx}} \omega_{\mathrm{ny}} k L_{\mathrm{n}} D_{\mathrm{n}}}\left[\left(k L_{\mathrm{B}}\right)^{-1}-\frac{\omega_{\mathrm{m}}}{\Omega_{\mathrm{i}}}\right],
\end{aligned}
$$

$$
\begin{aligned}
D_{\mathrm{yy}}= & -\omega_{\mathrm{m}}+\frac{\nu_{\mathrm{ne}} \nu_{\mathrm{in}} \omega_{\mathrm{A}}^{2}}{D_{\mathrm{n}} \Omega_{\mathrm{i}} \omega_{\mathrm{c}} \omega_{\mathrm{ny}} k L_{\mathrm{B}}} \\
& -\frac{\nu_{\mathrm{ne}} \omega_{\mathrm{A}}^{2}}{\Omega_{\mathrm{i}} \omega_{\mathrm{B}} k L_{\mathrm{B}}} D_{\mathrm{y}} \\
& +\frac{\omega_{\mathrm{A}}^{2}}{\omega_{\mathrm{B}}}-i \frac{k \Delta V}{\omega_{\mathrm{c}}} v_{\mathrm{in}}+\frac{\left(k c_{\mathrm{s}}\right)^{2}}{\omega_{\mathrm{c}}} \\
& +\frac{i v_{\mathrm{in}} \omega_{t}^{2} \nu_{\mathrm{ne}} \omega_{\mathrm{A}}^{2}}{\omega_{\mathrm{B}} \Omega_{\mathrm{i}} \omega_{\mathrm{cn}} \omega_{\mathrm{nx}} \omega_{\mathrm{ny}} k L_{\mathrm{n}} D_{\mathrm{n}}} .
\end{aligned}
$$

Further notations (few of them are repeated for convenience):

$$
\begin{aligned}
& \omega_{\mathrm{B}}=\omega+i k^{2} \eta_{\mathrm{ei}}-\frac{c^{2} k^{2}}{\omega_{\mathrm{pi}}^{2}} \frac{v_{\mathrm{in}} v_{\mathrm{ne}}}{\omega_{\mathrm{nx}} D_{\mathrm{n}}}, \quad \omega_{\mathrm{m}}=\omega_{\mathrm{c}}+i \nu_{\mathrm{in}}-\frac{v_{\mathrm{in}} \nu_{\mathrm{ni}}}{D_{\mathrm{n}} \omega_{\mathrm{nx}}}, \\
& \omega_{\mathrm{nx}}=\omega_{\mathrm{cn}}+i v_{\mathrm{ni}}-\frac{\omega_{\mathrm{t}}^{2}}{\left(k L_{\mathrm{n}}\right)^{2} \omega_{\mathrm{cn}}}, \quad \omega_{\mathrm{ny}}=\omega_{\mathrm{cn}}+i v_{\mathrm{ni}}-\frac{\omega_{\mathrm{t}}^{2}}{\omega_{\mathrm{cn}}}, \\
& \omega_{\mathrm{c}}=\omega-k V_{\mathrm{ioy}}, \omega_{\mathrm{cn}}=\omega-k V_{\mathrm{noy}}, \\
& D_{\mathrm{x}}=\frac{\nu_{\mathrm{in}}}{D_{\mathrm{n}} \omega_{\mathrm{nx}}}+i \frac{\Omega_{\mathrm{i}}}{\Omega_{\mathrm{e}}} \frac{\nu_{\mathrm{en}}}{v_{\mathrm{ne}}}, \quad D_{\mathrm{y}}=\frac{\nu_{\mathrm{in}}}{D_{\mathrm{n}} \omega_{\mathrm{ny}}}+i \frac{\Omega_{\mathrm{i}}}{\Omega_{\mathrm{e}}} \frac{\nu_{\mathrm{en}}}{v_{\mathrm{ne}}}, \\
& D_{\mathrm{n}}=1-\frac{\omega_{\mathrm{t}}^{4}}{\omega_{\mathrm{cn}}^{2} \omega_{\mathrm{nx}} \omega_{\mathrm{ny}}\left(k L_{\mathrm{n}}\right)^{2}}, \quad \Omega_{\mathrm{i}, \mathrm{e}}=\frac{e B_{0}}{m_{\mathrm{i}, \mathrm{e}} c}, \omega_{\mathrm{A}}=k V_{\mathrm{A}}, \\
& V_{\mathrm{A}}^{2}=\frac{B_{\mathrm{o}}^{2}}{4 \pi n_{\mathrm{io}} m_{\mathrm{i}}}, \quad \omega_{\mathrm{t}}=k V_{\mathrm{nt}}, \quad C_{\mathrm{s}}^{2}=\frac{T_{\mathrm{i}}}{m_{\mathrm{i}}}, \quad V_{\mathrm{nt}}^{2}=\frac{T_{\mathrm{n}}}{m_{\mathrm{n}}}, \\
& \Delta V=V_{\text {ioy }}-V_{\text {noy }}=-\frac{V_{\mathrm{A}}^{2}}{\Omega_{\mathrm{e}}} \frac{v_{\mathrm{en}}}{v_{\mathrm{in}}} \frac{1}{L_{\mathrm{B}}}=-\eta_{\mathrm{en}} \Omega_{\mathrm{i}} \frac{1}{L_{\mathrm{B}}}, \eta_{\mathrm{en}}=\frac{v_{\mathrm{en}} c^{2}}{\omega_{\mathrm{pe}}^{2}}, \\
& \omega_{\mathrm{pe}, \mathrm{pi}}=\left(\frac{4 \pi n e^{2}}{m_{\mathrm{e}, \mathrm{i}}}\right)^{1 / 2}, \quad L_{\mathrm{P}}^{-1}=\frac{\partial_{\mathrm{x}} n_{\mathrm{io}}(x)}{n_{\mathrm{io}}}, \\
& L_{\mathrm{n}}^{-1}=\frac{\partial_{\mathrm{x}} n_{\mathrm{no}}(x)}{n_{\mathrm{no}}}, \quad L_{\mathrm{B}}^{-1}=\frac{\partial_{\mathrm{x}} B_{\mathrm{io}}(x)}{B_{\mathrm{o}}} .
\end{aligned}
$$

\section{Appendix B}

The purpose of this Appendix is to provide better readability of the paper, otherwise Eqs. (B6) and (B7) are derivable from Eq. (A8).

\section{B1 Plasma momentum transfer equations}

\section{B1.1 X-component}

$$
\begin{aligned}
-i n_{\mathrm{io}} m_{\mathrm{i}} \omega_{\mathrm{c}} \tilde{V}_{\mathrm{ix}} & =-T \partial_{\mathrm{x}} \tilde{n}_{\mathrm{i}}-v_{\mathrm{eff}} n_{\mathrm{io}}\left(\tilde{V}_{\mathrm{ix}}-\tilde{V}_{\mathrm{nx}}\right) \\
& +\frac{i k_{\mathrm{y}} v_{\mathrm{en}} m_{\mathrm{e}} c}{4 \pi e} \tilde{B}
\end{aligned}
$$

where $v_{\text {eff }}=m_{\mathrm{e}} v_{\mathrm{en}}+m_{\mathrm{i}} v_{\mathrm{in}} \sim m_{\mathrm{i}} v_{\mathrm{in}}, C_{\mathrm{s}}^{2}=\frac{T}{m_{\mathrm{i}}} ; T=T_{\mathrm{i}}+T_{\mathrm{e}}$ and $v_{\text {ni }} \gg v_{\text {ne }}$.

\section{B1.2 Y-component}

$$
\begin{aligned}
-i n_{\mathrm{io}} m_{\mathrm{i}} \omega_{\mathrm{c}} \tilde{V}_{\mathrm{iy}} & =i k_{\mathrm{y}} n_{\mathrm{io}} \tilde{n}_{\mathrm{i}} T_{\mathrm{i}}-v_{\mathrm{eff}} n_{\mathrm{io}} \tilde{V}_{\mathrm{iy}} \\
& +v_{\mathrm{eff}} n_{\mathrm{io}}\left(\tilde{V}_{\mathrm{ny}}\right)-i k_{\mathrm{y}} \frac{B_{0} \tilde{B}}{4 \pi},
\end{aligned}
$$

we have used $v_{\text {in }} m_{\mathrm{i}} \gg m_{\mathrm{e}} v_{\mathrm{en}}$, and $v_{\mathrm{en}}=\frac{v_{\mathrm{ne}} m_{\mathrm{i}}}{m_{\mathrm{e}}}, \omega_{\text {ny }}=\omega_{\mathrm{cn}}+$ $i\left(v_{\mathrm{ni}}\right)-\left(\omega_{\mathrm{t}}^{2} / \omega_{\mathrm{cn}}\right)$.

\section{B2 Neutral momentum transfer equations}

\section{B2.1 X-component}

$\tilde{V}_{\mathrm{nx}}=\frac{i \nu_{\mathrm{ni}}}{\omega_{\mathrm{nx}}} \tilde{V}_{\mathrm{ix}}+\frac{k v_{\mathrm{ne}} c}{4 \pi n_{\mathrm{io}} e} \frac{\tilde{B}}{\omega_{\mathrm{nx}}} \hat{e}_{\mathrm{x}}$,

where $v_{\mathrm{ni}} \gg v_{\mathrm{ne}}$ and $\omega_{\mathrm{nx}}=\omega_{\mathrm{cn}}+i v_{\mathrm{ni}}$.

\section{B2.2 Y-component}

$\tilde{V}_{\text {ny }}=i \frac{v_{\text {ni }}}{\omega_{\text {ny }}} \tilde{V}_{\text {iy }}$

\section{B3 Magnetic field evolution}

$\frac{\tilde{B}_{\mathrm{Z}}}{B_{\mathrm{o}}}=\frac{i m_{\mathrm{i}} c k_{\mathrm{y}} \omega_{\mathrm{m} 1}}{e B_{0} \omega_{\mathrm{B} 1}} \tilde{V}_{\mathrm{ix}}+\frac{1}{\omega_{\mathrm{B} 1}} k \tilde{V}_{\mathrm{iy}}$,

where

$$
\begin{aligned}
& \omega_{\mathrm{B} 1}=\omega_{\mathrm{c}}+i k_{\mathrm{y}}^{2} \eta_{\mathrm{ei}}-\frac{c^{2} k^{2} v_{\mathrm{in}} \nu_{\mathrm{ne}}}{\omega_{\mathrm{pi}}^{2} \omega_{\mathrm{nx}}}, \\
& \omega_{\mathrm{m} 1}=\omega_{\mathrm{c}}+i \nu_{\mathrm{in}}-\frac{\nu_{\mathrm{in}} \nu_{\mathrm{ni}}}{\omega_{\mathrm{nx}}} .
\end{aligned}
$$

Solving these sets of equations for strongly coupled, cold and resistive PIP, we obtain following coupled equations:

$\tilde{V}_{\mathrm{ix}}\left(\frac{-i \omega}{\Omega_{i}^{*}}\right)+\left(\frac{\omega \omega_{\mathrm{B}}}{k^{2} V_{\mathrm{A}}^{* 2}}-1\right) \tilde{V}_{\mathrm{iy}}=0$,

$\tilde{V}_{\mathrm{ix}}\left(\frac{-i \omega \omega_{\mathrm{B}}}{k^{2} V_{\mathrm{A}}^{* 2}}-\frac{i \nu_{\text {ne }} \omega}{\Omega^{* 2}}\right)-\left(\frac{\nu_{\text {ne }}}{\Omega^{*}}\right) \tilde{V}_{\mathrm{iy}}=0$. 
Where we have used $v_{\text {ne }} v_{\text {in }}=\left(\frac{m_{\mathrm{e}}}{m_{\mathrm{i}}}\right) v_{\mathrm{en}} v_{\mathrm{ni}}$ and the recasted form of $\omega_{\mathrm{B}}=\omega_{\mathrm{c}}+i k^{2} \eta, \omega_{\mathrm{A}}^{*}=k V_{\mathrm{A}}^{*}, \eta=\eta_{\mathrm{ei}}+\eta_{\mathrm{en}}$.

Acknowledgements. We are grateful to Stan Cowley, who has read our manuscript and offered valuable suggestions for improvement of style of presentation. A. A. Shaikh expresses his sincere gratitude to Stan Cowley for support, encouragement and grant to carry out this research work. He thanks Theory Group of Physical Research laboratory, Ahemdabad, India, for using its facilities where this problem, partly, was formulated. Also the discussion with J. R. Bhatt is gratefully acknowledged. Finally, we are thankful to the two referees for useful comments which helped to improve this manuscript.

Topical Editor C. Owen thanks two anonymous referees for their help in evaluating this paper.

\section{References}

Alexandrov, A. F., Bogdankevich, L. S., and Rukhadaze, A. A.: Principles of Plasma Electrodynamics, Springer Series in Electrodynamics, vol. 9, Springer-Verlag Berlin Heidelberg, New York, 1984.

Amagishi, Y. and Tanaka, M.: Ion-neutral collision effect on an Alfvén wave, Phys. Rev. Lett., 71, 360-363,1993.

Andersson, L. and Ergun, R. E.: Neutral wind effects on ion outflow at Mars, Earth Planets Space (EPS), 64, 105-112, 2012.

Christopher, W. and Jeremy, H.: Alfvén Propagation in Partially ionized Plasma Phy. Plasma, 11, 1358-1365, 2004.
De Pontieu, B. and Haerendel, G.: Weakly Damped Alfvén Waves as Drivers for Spicules, Astron. Astrophys., 338, 729-736, 1998.

Huba, J. D.: Universal Interchange Instabilities in Partially Ionized Gases, Phys. Fluids, B2, 2547-2550, 1990.

Khodachenko, M. L., Arber, T. D., Rucker, H. O., and Hanslmeier, A.: Collisional and Viscous Damping of MHD waves in Partially Ionizsd of the Solar Atmosphere, Astron. Astrophys., 422, 10732550, 2004.

Pécseli, H. and Engvold, O.: Modeling of prominence threads in magnetic fields: levitation by incompressible MHD waves, Solar Physics, 194, 73-86, 2000.

Shaikh, D.: Dynamics of Alfvén waves in Partially ionized astrophysical plasmas, J. Plasma Physics, 76, 305-315, 2009.

Shaikh, A. A. and Das, A. C.: Does Neutral Dynamics suppress Instabilities in Partially Ionized Plasma?, J. Plasma Physics, 3, 173-190, 2002.

Shaikh, A. A., Das, A. C., and Agrawal, B. P.: Neutral dynamics and High frequency Electromagnetic Modes in partially ionized plasma, Physica Scripta, 59, 458-561, 1999.

Shukla, P. K.: Generation and dynamics of Plasma blobs in Partially ionized tokamak scrape-off-layer, Phys. Lett. A, 371, 453-456 2007.

Song, P. and Vasyliunas, M., L.: Solar wind-magnetosphereionosphere coupling: Neutral effects on signal propagation, J. Geophys. Res., 110, A09309, doi:10.1029/2005JA011139, 2005.

Song, P. and Vasyliunas, M. L.: Heating of the solar atmosphere by strong damping of Alfvén waves, J. Geophys. Res., 116, A09104, doi:10.1029/2011JA016679, 2011. 